\title{
artigo
}

\section{Enfermagem e a humanização da assistência em saúde mental: perspectivas e desafios}

\author{
Nursing and the humanization of mental health care: perspectives and challenges \\ Enfermería y humanización de la salud mental: perspectivas y desafíos
}

\begin{abstract}
RESUMO
Objetivo: Este estudo objetivou descrever a literatura recente sobre humanização da assistência de enfermagem na saúde mental. Método: trata-se de uma revisão integrativa de literatura realizada nas bases de dados Biblioteca Virtual de Saúde (BVS) e Web of Science em busca de publicações datadas entre 2011 e 2021. Resultados: Foram identificados oito artigos. As ações principais de humanização de enfermagem foram: Desenvolver escuta terapêutica e empática; estabelecer relação terapêutica; participar do reestabelecimento de cidadania e autonomia; e considerar as necessidades do núcleo familiar. As principais barreiras para a humanização da assistência identificadas foram: Prática automatizada e tecnicista; rede de atenção em saúde mental desarticulada; uso de violência; sobrecarga de trabalho; e preconceito. Conclusão: Considera-se que a enfermagem tem papel importante na construção do cuidado humanizado à pessoa com demandas de saúde mental e este cuidado deve ser construído com base na luta antimanicomial e no respeito à condição humana.
\end{abstract}

DESCRITORES: Enfermagem; Saúde Mental; Humanização da Assistência

\section{ABSTRACT}

Objective: This study aimed to describe recent literature on the humanization of nursing care in mental health. Method: this is an integrative literature review carried out in the Biblioteca Virtual de Saúde (BVS) and Web of Science databases in search of publications dated between 2011 and 2021. Results: Eight articles were identified. The main actions of humanization in nursing were: Develop therapeutic and empathic listening; establish a therapeutic relationship; participate in the reestablishment of citizenship and autonomy; and consider the needs of the household. The main barriers to the humanization of care identified were: Automated and technicist practice; disjointed mental health care network; use of violence; work overload; and prejudice. Conclusion: It is considered that nursing has an important role in the construction of humanized care for people with mental health demands and this care should be built based on the anti-asylum fight and respect for the human condition.

DESCRIPTORS: Nursing; Mental health; Humanization of Assistance.

\section{RESUMEN}

Objetivo: Este estudio tuvo como objetivo describir la literatura reciente sobre la humanización del cuidado de enfermería en salud mental. Método: se trata de una revisión integradora de la literatura realizada en las bases de datos Biblioteca Virtual de Saúde (BVS) y Web of Science en busca de publicaciones fechadas entre 2011 y 2021. Resultados: Se identificaron ocho artículos. Las principales acciones de humanización en enfermería fueron: la escucha terapéutica y empática; establecer relación terapéutica; participar en el restablecimiento de la autonomía; y considere las necesidades del hogar. Las principales barreras identificadas fueron: Práctica automatizada y tecnicista; red desarticulada de atención de salud mental; uso de la violencia; sobrecarga de trabajo; y prejuicio. Conclusión: Se considera que la enfermería tiene un papel importante en la construcción de un cuidado humanizado para pacientes de salud mental y este cuidado debe construirse a partir del respeto a la condición humana. DESCRIPTORES: Enfermería; Salud mental; Humanización de la assistência.

RECEBIDO EM: 08/06/21 APROVADO EM: 14/06/21

\section{Leandro Saldanha Nunes Mouzinho}

Enfermeiro. Psicólogo. Professor, Graduação em Enfermagem, Universidade CEUMA. São Luís (MA), Brasil. Graduação em Psicologia, Universidade Federal do Maranhão. São Luís (MA), Brasil. Mestre pelo Programa de Pós-Graduação em Saúde Coletiva pela Universidade Federal do Maranhão.

Instituição: Universidade Federal do Maranhão (UFMA).

ORCID: 0000-0001-5161-8212

\section{Antonio Carlos Garcês Alves Junior}

Economista e acadêmico de Psicologia. Graduação em Ciências Econômicas, Universidade Federal do Maranhão. São Luís (MA), Brasil. Graduando em Psicologia, UNDB Centro Universitário. São Luís (MA), Brasil. Especialista em Gestão Financeira e Controladoria pela Universidade Estácio de Sá. Instituição: UNDB Centro Universitário.

ORCID: 0000-0002-8269-0361 


\section{Cláudia Regina Nunes Eloi da Luz}

Médica. Professora, Graduação em Medicina, Universidade Federal do Maranhão, São Luís (MA), Brasil. Doutora pelo programa de Medicina pela Universidade Federal de São Paulo.

Instituição: Universidade Federal do Maranhão (UFMA).

ORCID: 0000-0002-3745-6605

\section{INTRODUÇÃO}

A enfermagem no campo da saúde mental traçou caminhos históricos de coerção e disseminação de práticas que cerceiam a liberdade das pessoas com demandas de saúde mental. A literatura traz diversos exemplos dessas práticas e de como a enfermagem tinha função de execução e controle, nem sempre com fins terapêuticos ou com planejamento terapêutico direcionado à melhora e independência desses indivíduos. 1-3

A literatura traz diversos exemplos de como a saúde mental tem fragilidades no quesito da humanização da assistência: seja considerando a estimativa de que há mais de 36 mil leitos psiquiátricos destinados a internação no Brasil atualmente; 4 a qualidade dos serviços da Rede de Atenção Psicossocial;5 resoluções do Conselho Federal de Enfermagem em busca de técnicas e cuidados mais humanizados;6,7 ou o interesse científico por estudar a reforma psiquiátrica na perspectiva dos cuidados de enfermagem. 8

Desde 2001, iniciou-se a reforma psiquiátrica no Brasil, encabeçada pela Lei 10.216, que marcou a redução gradual de leitos em hospitais psiquiátricos e manicômios pelo país e promoveu a legislação de direitos básicos para as pessoas que necessitam de atendimentos na saúde mental. Mesmo após muitos anos de luta antimanicomial, ainda há muitos desafios a serem superados e barreiras a serem transpostas, especialmente para a enfermagem.3

Uma dessas barreiras consiste na humanização da assistência nesses espaços de atendimento, visto que a enfermagem muitas vezes é designada a manejar clientes em crise e fica responsável por cuidados mais diretos a eles relacionados à autocuidado, alimentação e higiene.1-3 Além disso, há escassez de pesquisas científicas na área de enfermagem em saúde mental como um

\section{A literatura traz}

diversos exemplos dessas práticas e de como a enfermagem tinha função de execução e controle, nem sempre com fins terapêuticos ou com planejamento terapêutico

direcionado

à melhora e

independência desses indivíduos todo, como apontado por algumas revisões integrativas recentes.9-11

A partir do exposto, formulou-se a pergunta norteadora: "Quais as produções científicas da enfermagem sobre humanização da assistência em saúde mental no período de 2011 a 2021 ?”. Em busca de respostas para esse questionamento, este estudo teve como objetivo geral descrever a literatura recente sobre humanização da assistência de enfermagem na saúde mental e como objetivos específicos: a) Identificar a literatura recente sobre humanização da assistência de enfermagem na saúde mental; b) Elencar ações de humanização que o enfermeiro pode realizar na assistência na saúde mental; e c) Citar as barreiras para a humanização na assistência de enfermagem em saúde mental.

\section{MÉTODO}

Revisão integrativa de literatura referente à produção científica na temática proposta. Este método é desenvolvido em seis fases distintas, realizadas na pesquisa em questão na ordem a seguir: identificação do tema e elaboração da questão de pesquisa, amostragem ou pesquisa da literatura, colheita de dados, análise crítica dos estudos incluídos, interpretação e discussão dos resultados e apresentação da revisão/síntese de conhecimento. 12

A primeira etapa consiste em definir o problema de pesquisa, hipótese e questão norteadora; seguido da identificação das bases de dados a serem consultadas, descritores baseados no Descritores em Ciências da Saúde (DECS) e critérios de inclusão e exclusão; logo após, realiza-se a pesquisa nas bases de dados em busca das publicações que se adequem aos critérios estabelecidos, essa etapa também inclui a categorização dos estudos de acordo com critérios pré-estabelecidos de assunto / temática.12

As etapas correspondentes à análise crí- 


\section{artigo}

Mouzinho, L. S. N., Junior, A. C. G. A.,Luz, C. R. N. E.

Enfermagem e a humanização da assistência em saúde mental: perspectivas e desafios

tica dos estudos e à interpretação e discussão dos resultados são realizadas com base na identificação dos assuntos coincidentes e na análise das divergências; também é realizada a discussão com base na literatura recente para trazer novas perspectivas ao tema. Por fim, a síntese das análises é organizada e disposta no formato de quadros, gráficos e discussões sistematizadas. 12

A seleção dos artigos ocorreu na primeira quinzena de setembro de 2021. Neste período, foram selecionados artigos publicados e indexados nas bases de dados Web of Science e Biblioteca Virtual em Saúde (BVS), através dos descritores: "enfermagem", "saúde mental" e "humanização"; ou seus equivalentes em inglês "nursing", "mental health" e "humanization". Os critérios de inclusão foram: Artigos originais; oriundos de método de pesquisa de campo exploratória qualitativa ou quantitativa; disponíveis na íntegra em periódicos nacionais ou internacionais; publicados em português, inglês, francês ou espanhol no período de 2011 a 2021. Não foram incluídos estudos em que outros profissionais da equipe multiprofissional participaram da coleta de dados sem especificar os participantes enfermeiros separadamente nos resultados.

Para definição da amostra, os títulos e informações gerais dos artigos foram lidos nas bases de indexação em busca dos descritores e dos critérios de inclusão, preliminarmente. A segunda etapa consistiu na leitura dos resumos em busca de inadequações aos critérios apresentados e, por último, foi realizada leitura do texto na íntegra. Após todas as etapas, foi definida a amostra final de artigos para análise. Todas as etapas foram realizadas por dois pesquisadores e as amostras foram comparadas para definição do quantitativo final de publicações.

Para análise dos dados, foi utilizado o método da Análise de Conteúdo, que preconiza a organização das informações em categorias distintas ou afins, que permitem a análise do conteúdo identificado. Essa classificação ocorre a partir de três fases: Pré-análise, exploração do material e tratamento dos resultados.13

Para o tratamento dos resultados, foram analisados os conteúdos das publicações em busca de coincidências e divergências entre as informações encontradas e foi possível fazer considerações sobre as perspectivas e desafios que o enfermeiro precisa enfrentar na humanização da sua assistência em saúde mental.

\section{RESULTADOS}

Foram encontrados, ao todo, oito artigos de acordo com os critérios relatados. Na plataforma BVS, a primeira busca resultou em 96 resultados, destes apenas seis se enquadraram nos critérios escolhidos para

\section{Quadro 01: Identificação dos artigos sobre humanização da assistência em enfermagem na saúde mental publicados entre os anos de 2011 a 2021.}

\begin{tabular}{|c|c|c|c|}
\hline $\begin{array}{l}\text { BASE } \\
\text { DE } \\
\text { DADOS }\end{array}$ & AUTOR & ANO & TÍTULO \\
\hline BVS & $\begin{array}{lr}\text { Oliveira } & \text { LC, } \\
\text { Silva RAR, } \\
\text { Medeiros MN } \\
\text { et al. }\end{array}$ & 2015 & $\begin{array}{l}\text { Cuidar humanizado: descobrindo as possibilidades na } \\
\text { prática da enfermagem em saúde mental }\end{array}$ \\
\hline BVS & $\begin{array}{l}\text { Lima GZ, } \\
\text { Feltrin JÁ, } \\
\text { Rodrigues J J } \\
\text { et al. }\end{array}$ & 2016 & $\begin{array}{l}\text { Perception of nursing students on mental health } \\
\text { home-care: a qualitative approach }\end{array}$ \\
\hline BVS & $\begin{array}{l}\text { Moll MF, Men- } \\
\text { des AC, Ventu- } \\
\text { ra CAA et al. }\end{array}$ & 2016 & $\begin{array}{l}\text { Os cuidados de enfermagem e o exercício dos direitos } \\
\text { humanos: Uma análise a partir de realidade em Por- } \\
\text { tugal }\end{array}$ \\
\hline BVS & $\begin{array}{l}\text { Oliveira LC, } \\
\text { Menezes HF, } \\
\text { Oliveira RL et } \\
\text { al. }\end{array}$ & 2018 & $\begin{array}{l}\text { Atendimento móvel às urgências e emergências psi- } \\
\text { quiátricas: percepção de trabalhadores de enferma- } \\
\text { gem }\end{array}$ \\
\hline BVS & $\begin{array}{l}\text { Guimarães } \\
\text { JCS, Santos } \\
\text { BL, Aperiben- } \\
\text { se PGGS et al. }\end{array}$ & 2018 & $\begin{array}{l}\text { Eletroconvulsoterapia: construção histórica do cuida- } \\
\text { do de Enfermagem (1989-2002) }\end{array}$ \\
\hline BVS & $\begin{array}{l}\text { Lima DWC, } \\
\text { Paixão AKR, } \\
\text { Bezerra KP et } \\
\text { al. }\end{array}$ & 2021 & $\begin{array}{l}\text { Humanização no cuidado em saúde mental: compre- } \\
\text { ensões dos enfermeiros }\end{array}$ \\
\hline $\begin{array}{l}\text { Web of } \\
\text { Science }\end{array}$ & $\begin{array}{l}\text { Thifault MC, } \\
\text { Kirouac L }\end{array}$ & 2019 & $\begin{array}{l}\text { Les infirmières psychiatriques témoins d'un mouve- } \\
\text { ment d'humanisation au cours des premières et deu- } \\
\text { xièmes vagues de la désinstitutionnalisation au Qué- } \\
\text { bec (1960-1990) }\end{array}$ \\
\hline $\begin{array}{l}\text { Web of } \\
\text { Science }\end{array}$ & $\begin{array}{l}\text { Silva PMC, } \\
\text { Costa NF, Bar- } \\
\text { ros DRRE et al. }\end{array}$ & 2019 & $\begin{array}{l}\text { Saúde mental na atenção básica: possibilidades e fra- } \\
\text { gilidades do acolhimento }\end{array}$ \\
\hline
\end{tabular}

seleção dos artigos. Já na plataforma Web of Science, a primeira busca resultou em 16 resultados, finalizando com três artigos que estavam incluídos nos critérios. Após busca por publicações repetidas nas amostras escolhidas, houve a exclusão de uma obra, finalizando com o total de oito artigos participantes da amostra final. As informações relativas às publicações analisadas podem ser verificadas no quadro 01 .

Quanto ao método dos artigos, sete dos oito encontrados utilizaram a abordagem exploratória qualitativa14-20 e um utilizou método descritivo observacional.21 As ações de humanização mais identificadas 
foram: Desenvolver escuta terapêutica e empática;14,15,17-20 estabelecer relação terapêutica;14,15,17,18,21 participar do restabelecimento de cidadania e autonomia;14,17,18,21 e considerar as necessidades do núcleo familiar.14,18

Sobre as barreiras identificadas, podem ser elencadas: Prática de enfermagem automatizada e baseada em técnicas;15-18 falta de rede de atenção em saúde mental integrada e articulada; 15,20 uso de violência na assistência; 15,16 sobrecarga de trabalho na enfermagem;19 e preconceito com pessoas que têm demandas de saúde mental.14

\section{DISCUSSÃO}

Outras revisões integrativas com a mesma temática também encontraram resultados semelhantes aos descritos, tanto pela maioria dos artigos identificados utilizarem a abordagem exploratória qualitativa para coleta de dados quanto ao destaque para o desenvolvimento de novas abordagens em saúde mental, com respeito aos princípios da reforma psiquiátrica e o estabelecimento da relação terapêutica e de incentivo à autonomia do usuário.9-11

A integração da Rede de Atenção Psicossocial foi um achado relevante coincidente a uma outra revisão integrativa que buscou identificar o cuidado humanizado multiprofissional e identificou os Centros de Atenção Psicossociais (CAPS) como ferramentas de cuidado humanizado comunitário e fortes aliados no desenvolvimento de cidadania e autonomia tanto para os seus usuários quanto para suas famílias. 22

Sobre os desafios, poucas revisões buscaram identificar explicitamente as barreiras para a humanização do processo, porém é comum identificar relatos quanto a automatização de rotinas de enfermagem pouco terapêuticas, que não priorizam o desenvolvimento do cuidar integral e a autonomia da pessoa. 9,11

Os resultados encontrados relacionados às ações de enfermagem humanizadas já são cuidados preconizados pela literatura especializada na área. O estabelecimento da relação terapêutica, realização de escuta empática e especializada, cuidados familiares e incentivo a autonomia do sujeito são frequentemente mencionados no desenvolvimento de planos de cuidados de enfermagem na saúde mental.1-3

Este achado da literatura especializada contrasta com o resultado encontrado na pesquisa atual das barreiras ao desenvolvimento do cuidado humanizado, visto que a

\section{Outro achado}

\section{que é identificado}

pela literatura

recente consiste

na sobrecarga

do trabalho de

enfermagem e como

esta pode ser um

agravante para 0

desenvolvimento

de práticas

desumanizadas na

assistência [...]

prática tecnicista-automatizada e o uso da violência na terapêutica não deveriam ser questões presentes se os enfermeiros estivessem seguindo as recomendações de cuidados humanizados já preconizados.

Essa discussão traz questões relacionadas à formação e educação continuada de en- fermeiros que trabalham na saúde mental. Alguns autores já apontam em estudos recentes como a área da enfermagem em saúde mental carece de iniciativas de formação continuada para que seus cuidados sejam baseados em evidência e nos preceitos da ética e da luta antimanicomial, o que pode ser uma causa da continuidade de práticas pouco humanizadas e manicomiais.23,24

Outro achado que é identificado pela literatura recente consiste na sobrecarga do trabalho de enfermagem e como esta pode ser um agravante para o desenvolvimento de práticas desumanizadas na assistência,25,26 inclusive na própria área da saúde mental, espaço em que o profissional precisa lidar também com questões inter e intrapessoais específicas como a dificuldade de gestão de serviços de saúde mental e o próprio conceito de trabalhar com a "loucura".27

As dificuldades mencionadas no parágrafo acima incluem também a falta de integração entre os serviços na Rede de Atenção Psicossocial, um dos achados desta pesquisa quanto às barreiras às ações de humanização. Esta questão em específico já é uma dificuldade relatada há décadas para a adequada integralidade do serviço, e é uma questão que emergiu e merece maior atenção desde o ano de 2016, em que há claros retrocessos a nível de políticas públicas em saúde mental que distanciam do cuidar integral, comunitário e antimanicomial.28

\section{CONCLUSÕES}

O cuidado humanizado em enfermagem na área de saúde mental é uma temática de grande importância visto o histórico da psiquiatria e a função que esta classe já desempenhou nos manicômios. Portanto, deve ser sempre um cuidado, tanto clínico quanto de investimento científico, o desenvolvimento de tecnologias e de investigações sobre o tema.

O estudo atual tem suas potencialidades no desenvolvimento de um tema de grande relevância para a área da saúde mental e enfermagem e no escopo de coleta de dados, trazendo artigos nacionais e internacionais inclusive em línguas pouco exploradas por 


\section{artigo}

Mouzinho, L. S. N., Junior, A. C. G. A., Luz, C. R. N. E.

Enfermagem e a humanização da assistência em saúde mental: perspectivas e desafios

outras revisões já realizadas. Ao final deste estudo, pode-se considerar que foi possível realizar o levantamento das pesquisas recentes sobre a humanização da assistência em enfermagem na saúde mental no período de 2011 a 2021, além de trazer discussões relevantes sobre esta temática.

As limitações, e aqui colocadas já como indicações para continuidade do desenvol- vimento científico da temática, consistem na delimitação de ações de humanização no campo da enfermagem em saúde mental. O desenvolvimento de pesquisas na área da saúde mental primando pela visão multiprofissional é diversa e pode trazer perspectivas diferentes sobre o cuidar generalista, o que vai além dos objetivos da atual pesquisa.
O compromisso com a luta antimanicomial e com os seus preceitos de assistência humanizada devem ser objetivos diários para quaisquer profissionais que trabalham na saúde mental, em destaque à enfermagem que lida com as necessidades de saúde do sujeito e de como este se insere nos seus vínculos sociais, familiares e intrapessoais.

\section{REFERÊNCIAS}

1 Townsend MC, Morgan KI. Psychiatric mental health nursing: concepts of care in evidence-based practice. $9^{\circ}$ ed. Philadelphia, PA: F.A. Davis Company; 2018

2 Videbeck SL. Psychiatric-mental health nursing. $8^{\circ}$ ed. Philadelphia, PA: Wolters Kluwer; 2020.

3 Fukuda IMK, Stefanelli MC, Arantes EC. Enfermagem psiquiátrica: em suas dimensões assistenciais. $2^{\circ}$ ed. São Paulo: Manole; 2017.

4 Conselho Federal de Psicologia. Hospitais psiquiátricos no Brasil: relatório de inspeção nacional. $2^{\circ}$ ed. Brasil: Conselho Federal de Psicologia; 2020

5 Barcelos MV, Teixeira ER, Santana PPC. Satisfação com os serviços dos centros de atenção psicossocial: uma revisão integrativa. Saúde Coletiva (Barueri). 2021;11(68):7827-7836.

6 Conselho Federal de Enfermagem. Resolução COFEN N 427/2012. Normatiza os procedimentos da enfermagem no emprego de contenção mecânica de pacientes. Diário Oficial da União. 07 mai. 2012;90(176 seção 1):176.

7 Conselho Federal de Enfermagem. Resolução COFEN Nº 678/2021. Aprova a atuação da Equipe de Enfermagem em Saúde Mental e em Enfermagem Psiquiátrica. Diário Oficial da União. 19 dez. 2018;245(148 seção 1):899.

8 Silva JS, Ribeiro HKP, Fernandes MA, Rocha DM. O cuidar de enfermagem em saúde mental na perspectiva da reforma psiquiátrica. Enferm. Foco. 2020:11(1):170-175

9 Júnior JMP, Clementino FS, Santos RCA, Vitor AF, Miranda FAN. Enfermagem e o processo de desinstitucionalização no âmbito da saúde mental revisão integrativa. Rev Fund Care Online. 2017;9(3):893-898.

10 Silva PO, Silva DVA, Rodrigues CAO, Santos NHF, Barbosa SFA, Souto VD et al. Cuidado clínico de enfermagem em saúde mental. Rev enferm UFPE online. 2018:12(11):3133-46.

11 Santos BM, Silva RMCRA, Pereira ER, Joaquim FL, Goés TRP. A percepção dos estudantes de enfermagem sobre o cuidado humanizado: revisão integrativa. Rev Bras Enferm [Internet]. 2018;71(suppl 6):2965-73.

12 Sousa LMM, Marques-vieira CMA, Severino SSP, Antunes AV. A metodologia de revisão integrativa da literatura em enfermagem. Revista Investigação em Enfermagem. 2017;2(21):17-26.

13 Cardoso MRG, Oliveira GS, Ghelli KGM. Análise de conteúdo: uma metodologia de pesquisa qualitativa. Cadernos da Fucamp. 2021;20(43):98-111.

14 Lima GZ, Feltrin JA, Rodrigues JJ, Buriola AA. Perception of nursing students on mental health home-care: a qualitative approach. $R$. pesq. cuid fundam. online [Internet]. 2016;8(2):4255-68.

15 Oliveira LC, Menezes HF, Oliveira RL, Lima DM, Fernandes SF, Silva RAR. Atendimento móvel às urgências e emergências psiquiátricas: percepção de trabalhadores de enfermagem. Rev. Bras. Enferm. 2020;73(1):e20180214.

16 Guimarães JCS, Santos BL, Aparibense GGS, Martins GCS, Peres AA, Santos TCF. Eletroconvulsoterapia: construção histórica do cuidado de Enfermagem (1989-2002). Rev. Bras. Enferm. 2018;71(suppl 6):2743-2750.

17 Lima DWC, Paixão AKR, Bezerra KPF, Azevedo RJMA, Sousa LD, Rosado FR. Humanização no cuidado em saúde mental: compreensões dos enfermeiros. SMAD. Revista eletrônica saúde mental álcool e drogas. 2021;17(1):58-65

18 Oliveira LC, Silva RAR, Medeiros MN, Queiroz JC, Guimarães J. Cuidar humanizado: descobrindo as possibilidades na prática da enfermagem em saúde mental. Rev. Pesqui. (Univ. Fed. Estado Rio J., Online). 2015;7(1):1774-1782.

19 Silva PMC, Costa NF, Barros DRR, Silva SJJ, Silva JFRL, Silva BT. Saúde mental na atenção básica: possibilidades e fragilidades do acolhimento. Rev Cuid [Internet]. 2019;10(1):e617.

20 Thifault MC, Kirouac, L. Les infirmières psychiatriques témoins d'un mouvement d'humanisation au cours des premières et deuxièmes vagues de la désinstitutionnalisation au Québec (1960-1990). Recherche en soins infirmiers. 2019;139(4):99-108.

21 Moll MF, Mendes AA. Os cuidados de enfermagem e o exercício dos direitos humanos: uma análise a partir de realidade em Portugal. Esc. Anna Nery. 2016;20(2):236-242

22 Araujo VSC, Souza LO, Duarte KO, Pereira RMO, Almeira LS, Reis MH et al. O desempenho exercido no processo de humanização da saúde mental: uma revisão integrativa. Revista Eletrônica Acervo Saúde. 2020;53(e3642):1-9.

23 Santos RGF, Nascimento JL. Panorama da educação continuada em enfermagem: conseqüências à qualificação do trabalho de enfermagem em saúde mental e psiquiatria. Revista Saúde e Desenvolvimento. 2017:11(7):1-15.

24 Scafuto JCB, Saraceno B, Delgado PGG. Formação e educação permanente em saúde mental na perspectiva da desinstitucionalização (20032015). Com. Ciências Saúde. 2017;28(3/4):350-358.

25 Gomes, RM. Humanização e desumanização no trabalho em saúde. Rio de Janeiro: Fiocruz; 2017.

26 Muniz DC, Andrade EGS, Santos WLS. A saúde do enfermeiro com a sobrecarga de trabalho. Rev Inic Cient Ext. 2019;2(Esp.2):274-9.

27 Bellenzani R, Paro DM, Oliveira MC. Trabalho em Saúde Mental e Estresse na Equipe: Questões para a Política Nacional de Humanização/SUS. Revista Psicologia e Saúde. 2016;8(1):32-43.

28 Cruz NFO, Gonçalves RW, Delgado PGG. Retrocesso da Reforma Psiquiátrica: o desmonte da política nacional de saúde mental brasileira de 2016 a 2019. Trabalho, Educação e Saúde. 2020;18(3):e00285117. 